\title{
Robust Stability Analysis of Fuzzy Feedback Linearization Control Systems
}

\author{
Chang-Woo Park*, Chang-Hoon Lee ${ }^{* * *}$ and Mignon Park* \\ *Dept. of Electrical and Electronic Eng., Yonsei Univ. \\ 134, Shinchon-dong, Seodaemun-ku, Seoul, Korea \\ **Division of Information and Communication Engineering, Paichai University \\ 436-9 Doma-dong, Seo-gu, Taejon 302-735, Korea
}

\begin{abstract}
In this paper, we have studied a numerical stability analysis method for the robust fuzzy feedback linearization regulator using Takagi-Sugeno fuzzy model. To analyze the robust stability, we assume that uncertainty is included in the model structure with known bounds. For these structured uncertainty, the robust stability of the closed system is analyzed by applying Linear Matrix Inequalities theory following a transformation of the closed loop systems into Lur'e systems.
\end{abstract}

Key words : Takagi-Sugeno fuzzy model, feedback linearization, linear matrix inequalities, robust scontrol, fuzzy cointrol

\section{I . Introduction}

Fuzzy feedback linearization is a feedback linearization method which uses a fuzzy model as a nonlinear system model. In [3], the fuzzy feedback linearization concept was introduced using Takagi-Sugeno fuzzy model. However, robustness issue which is significant in practical applications was not considered in this work. In some previous researches, adaptive techniques were applied [4]-[7]. While adaptive fuzzy feedback linearization guarantees Lyapunov stability in the presence of uncertainty, it has some practical limitations because of its complex structures.

LMI theory is the new and fast growing field and an valuable alternative to the analytical method [10], [11]. A variety of problems arising in system and control theory can be reduced to a few standard convex or quasiconvex optimization problems involving LMI. Since these resulting optimization problems can be easily solved by numerical computation, LMI techniques are very efficient and practical tools for the complex control problems. Specifically, a class of fuzzy control problems which is difficult to solve analytically, LMI techniques can afford the practical solutions. In the recent papers [12]-[16], the applicability of LMI techniques were showed excellently to fuzzy control systems.

To apply LMI techniques to our stability analysis problems, the closed system should be transformed into the standard form which has available LMI solution. In this paper, a numerical robust stability analysis for the fuzzy feedback linearization regulator is presented using Linear Matrix Inequalities (LMI) Theory. Well-known Takagi-Sugeno fuzzy model is used as the nonlinear plant model. Uncertainty is assumed to be included in the model structure with known bounds. For these structured uncertainty, the closed system can

Manuscript received January 14, 2002 ; revised February 20. 2002 be cast into Lur'e system by simple transformation. From the LMI stability condition for Lur'e system [17], we can derive the robust stability condition for the fuzzy feedback linearization regulator based on Takagi-Sugeno fuzzy model.

\section{The fuzzy feedback linearization regulator based on T-S fuzzy model}

\subsection{Problem formulation}

Consider the regulation problem of the following $n$-th order nonlinear SISO system

$$
x^{(n)}=f(x)+g(x) u
$$

where $f$ and $g$ are unknown or uncertain, but bounded continuous nonlinear functions. Let $x=\left[x, \dot{x}, \cdots, x^{(n-1)}\right]^{T}$ $\in R^{n}$ be the state vector of the system which is assumed to be available.

In this paper, well-known Takagi-Sugeno fuzzy model is used to identify the unknown nonlinear system (1). Takagi-Sugeno fuzzy mode is available in IF-THEN form (2) or Input-Output form (3).

\section{- IF-THEN form}

plant rule $i$ :

$$
\begin{aligned}
& \text { IF } x \text { is } M_{i 1} \text { and } \dot{x} \text { is } M_{i 2} \text { and } \cdots \text { and } x^{(n-1)} \text { is } M_{i n} \\
& \begin{array}{c}
\text { THEN } x^{(n)}=\left(\boldsymbol{a}_{i}+\Delta \boldsymbol{a}_{i}(t)\right)^{T} \cdot x+\left(b_{i}+\Delta b_{i}(t)\right) u, \\
i=1,2, \cdots, r
\end{array} \\
& \text { where } x=\left[x, \dot{x}, \cdots, x^{(n-1)}\right]^{T}, \\
& \qquad a_{i}, \Delta a_{i}(t) \in R^{1 \times n}, b_{i}, \Delta b_{i}(t) \in R
\end{aligned}
$$

In (2), $M_{i j}$ is the fuzzy set and $r$ is the number of rules. Also, $\Delta a_{i}(t)$ and $\Delta b_{i}(t)$ denotes the norm-bounded time- 
varying modeling uncertainty.

\section{- Input-Output form}

$$
\begin{aligned}
x^{(n)} & =\frac{\sum_{i=1}^{r} w_{i}(\boldsymbol{x})\left\{\left(\boldsymbol{a}_{i}+\Delta \boldsymbol{a}_{i}(t)\right)^{T} \cdot \boldsymbol{x}+\left(b_{i}+\Delta b_{i}(t)\right) u\right\}}{\sum_{i=1}^{r} w_{i}(\boldsymbol{x})} \\
& =\sum_{i=1}^{r} h_{i}(\boldsymbol{x})\left\{\left(\boldsymbol{a}_{i}+\Delta \boldsymbol{a}_{i}(t)\right)^{T} \cdot \boldsymbol{x}+\left(b_{i}+\Delta b_{i}(t)\right) u\right\}
\end{aligned}
$$

where

$$
w_{i}(\boldsymbol{x})=\prod_{j=1}^{n} M_{i j}\left(x^{(j-1)}\right), \quad h_{i}(\boldsymbol{x})=\frac{w_{i}(\boldsymbol{x})}{\sum_{i=1}^{x} w_{i}(\boldsymbol{x})}
$$

$M_{i j}\left(x^{(j-1)}\right)$ is the grade of membership of $x^{(j-1)}$ in $M_{i j}$. It is assumed in this paper that

$$
w_{i}(\boldsymbol{x}) \geq 0, i=1,2, \cdots, r, \sum_{i=1}^{r} w_{i}(\boldsymbol{x})>0
$$

Therefore,

$$
h_{i}(x) \geq 0, i=1,2, \cdots, r, \quad \sum_{i=1}^{k} h_{i}(x)=1
$$

For (3) to be controllable, $\sum_{i=1}^{k} h_{i}(x) b_{i} \neq 0$ for $x$ in certain controllability region $U_{c} \subset R^{n}$ is required. If this controllability requirement is satisfied, the following fuzzy feedback linearization regulator (4) can cancel the nonlinearity of (3) and achieve perfect linearization (5).

$$
\begin{aligned}
u & =\frac{\hat{\boldsymbol{a}}^{T} \cdot \boldsymbol{x}-\sum_{i=1}^{r} h_{i}(\boldsymbol{x}) \boldsymbol{a}_{i}{ }^{T} \cdot \boldsymbol{x}}{\sum_{i=1}^{+} h_{i}(\boldsymbol{x}) b_{i}} \\
& =\frac{\sum_{i=1}^{\gamma} h_{i}(x)\left(\hat{\boldsymbol{a}}^{T}-\boldsymbol{a}_{i}{ }^{T}\right) \cdot \boldsymbol{x}}{\sum_{i=1}^{r} h_{i}(\boldsymbol{x}) b_{i}}
\end{aligned}
$$

where we use the same $a_{i}, b_{i}$ and $h_{i}(x)$ with the fuzzy model (3) for all $i$ and $\widehat{a} \in R^{1 \times n}$ is the linear state feedback gain vector. The perfectly linearized system can be written as (5).

$$
x^{(n)}=\widehat{a}^{T} \cdot x
$$

However, due to the inevitable uncertainty, perfect linearization can not be achieved in practical application. By substituting (4) into (3), the imperfectly linearized system can be written as (6). From the bounds of $\Delta a_{i}(t)$ and $\Delta b_{i}(t)$, the bound of $a_{N j}(t)$ can be derived as in Appendix A. Thus, the closed system (6) can be treated as the linear system with the sector bounded nonlinearities. In the next section, the numerical robust stability analysis via LMI for the closed system (6) will be presented.

$$
\begin{gathered}
x^{(n)}=\widehat{a}^{T} \cdot x+\sum_{i=1}^{r} h_{i}(x) \Delta a_{i}(t)^{T} \cdot x \\
+\frac{\sum_{i=1}^{r} h_{i}(x) \Delta b_{i}(t)}{\sum_{i=1}^{k} h_{i}(x) b_{i}}\left\{\sum_{i=1}^{k} h_{i}(x)\left(\widehat{a}-a_{i}\right) \cdot x\right\}
\end{gathered}
$$

$$
=\widehat{\boldsymbol{a}}^{T} \cdot \boldsymbol{x}+\boldsymbol{a}_{N}(t)^{T} \cdot \boldsymbol{x}
$$

where

$$
\begin{gathered}
\boldsymbol{a}_{N}(t)=\sum_{i=1}^{x} h_{i}(x) \Delta a_{i}(t)^{T} \cdot x \\
+\frac{\sum_{i=1}^{x} h_{i}(x) \Delta b_{i}(t)}{\sum_{i=1}^{x} h_{i}(x) b_{i}}\left\{\sum_{i=1}^{x} h_{i}(x)\left(\widehat{a}-a_{i}\right)^{T} \cdot x\right\}
\end{gathered}
$$

\subsection{Implementation}

To implement the fuzzy feedback linearization regulator (4) in the fuzzy rule-based form, we utilize the control structure shown in Figure 1, which is the same one used in [8]. In this structure, the fuzzy feedback linearization regulator is divided into two blocks, a fuzzy rule-based controller block and a simple nonlinear function block. The fuzzy controller block shares the same fuzzy sets $M_{i j}$ and the parameters $a_{i}$ and $b_{i}$ with the fuzzy model in the premise parts for all $i$ and $j$. Therefore, $\hat{a}$ is the only design parameter of the fuzzy controller block. The nonlinear function block simply divides $u_{1}$ by $u_{2}$ to produce the same $u$ as in Eqn. (4). $i$-th rule of the fuzzy controller block can be represented by (7).

$$
\begin{gathered}
\text { IF } x \text { is } M_{i 1} \text { and } \dot{x} \text { is } M_{i 2} \text { and } \cdots \text { and } x^{(n-1)} \text { is } M_{i n} \\
\text { THEN }\left[\begin{array}{l}
u_{1} \\
u_{2}
\end{array}\right]=\left[\begin{array}{c}
\left(\widehat{a}-a_{i}\right)^{T} \cdot x \\
b_{i}
\end{array}\right], i=1,2, \cdots, r
\end{gathered}
$$

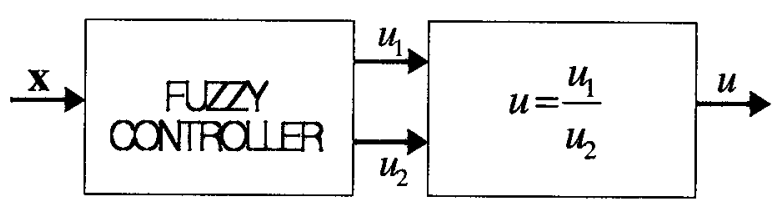

Fig. 1. Control structure of fuzzy feedback linearization regulator

\section{LMI-based robust stability analysis}

Consider the following Lur'e system (8)

$$
\begin{gathered}
\dot{x}=A x+B p \\
p_{i}(t)=\phi_{i}\left(x_{i}(t)\right), \quad i=1, \cdots, n_{p}
\end{gathered}
$$

where $p(t) \in R^{n_{\rho}}$, and the functions $\phi_{i}$ satisfy the [0, 1] sector conditions

$$
0 \leq \sigma \phi_{i}(\sigma) \leq \sigma^{2} \quad \text { for all } \sigma \in R
$$

or equivalently,

$$
\phi_{i}(\sigma)\left(\phi_{i}(\sigma)-\sigma\right) \leq 0 \quad \text { for all } \sigma \in R
$$

The linear system with the sector bounded nonlinearities can be cast into Lur'e system. Therefore, the closed system (6) 
can be cast into Lur'e system. In Theorem 1, Lyapunov stability condition for Lur'e system is derived using LMI Theory. In the proof of Theorem 1, S-procedure in LMI techniques [10] is used.

Theorem 1: Lur'e system (8) is stable in the sense of Lyapunov if there exist $\boldsymbol{P}>\mathbf{0}$,

$$
\begin{aligned}
& \boldsymbol{\Lambda}=\operatorname{diag}\left(\lambda_{1}, \cdots, \lambda_{n_{p}}\right) \geq 0 \text { and } \\
& \boldsymbol{T}=\operatorname{diag}\left(\tau_{1}, \cdots, \tau_{n_{p}}\right) \geq 0 \text { which satisfy LMI (10). } \\
& {\left[\begin{array}{cc}
\boldsymbol{A}^{T} \boldsymbol{P}+\boldsymbol{P} \boldsymbol{P} & \boldsymbol{P B}+\boldsymbol{A}^{T} \boldsymbol{\Lambda}+\boldsymbol{T} \\
\boldsymbol{B}^{T} \boldsymbol{P}+\boldsymbol{A}+\boldsymbol{T} & \boldsymbol{A} \boldsymbol{B}+\boldsymbol{B}^{T} \boldsymbol{\Lambda}-2 \boldsymbol{T}
\end{array}\right]<0}
\end{aligned}
$$

Proof : Let us choose a Lyapunov function

$$
V(\boldsymbol{x})=\boldsymbol{x}^{\boldsymbol{T}} \boldsymbol{P} \boldsymbol{x}+2 \sum_{i=1}^{n_{\boldsymbol{k}}} \lambda_{i} \int_{0}^{x_{i}} \phi_{i}(\sigma) d \sigma
$$

Thus the data describing the Lyapunov function are the matrix $\boldsymbol{P}$ and the scalars $\lambda_{i}, i=1, \cdots, n_{p}$. For $V(x)$ to be positive for nonzero $x$, we require

$$
P>0 \text { and } \Lambda=\operatorname{diag}\left(\lambda_{1}, \cdots, \lambda_{n_{p}}\right) \geq 0 \text {. }
$$

The time derivative of $V(x)$ is

$$
\frac{d V(\boldsymbol{x})}{d t}=2\left(\boldsymbol{x}^{\boldsymbol{T}} \boldsymbol{P}+\sum_{i=1}^{n_{1}} \lambda_{i} p_{i} \boldsymbol{I}_{i}\right)(\boldsymbol{A} \boldsymbol{x}+\boldsymbol{B p})
$$

where $I_{i}$ denotes the $i$-th row of $n \times n$ identity matrix.

Lyapunov stability condition $\frac{d V(x)}{d t}<0$ holds for all nonzero $x$ if and only if

$$
\left(\boldsymbol{x}^{T} \boldsymbol{P}+\sum_{i=1}^{n_{n}} \lambda_{i} p_{i} I_{i}\right)(A x+B p)<0
$$

for all nonzero $x$.

The $S$-procedure in LMI techniques then yields the following LMI condition

$$
\left[\begin{array}{cc}
A^{T} \boldsymbol{P}+\boldsymbol{P A} & \boldsymbol{P B}+\boldsymbol{A}^{T} \boldsymbol{\Lambda}+\boldsymbol{T} \\
\boldsymbol{B}^{T} \boldsymbol{P}+\boldsymbol{\Lambda} \boldsymbol{A}+\boldsymbol{T} & \boldsymbol{\Lambda} \boldsymbol{B}+\boldsymbol{B}^{T} \boldsymbol{\Lambda}-2 \boldsymbol{T}
\end{array}\right]<0
$$

where

$$
\begin{aligned}
& \boldsymbol{T}=\operatorname{diag}\left(\tau_{1}, \cdots, \tau_{n_{p}}\right) \geq 0, \\
& \Lambda=\operatorname{diag}\left(\lambda_{1}, \cdots, \lambda_{n_{p}}\right) \geq 0 .
\end{aligned}
$$

Therefore, Lur'e system (8) is stable in the sense of Lyapunov if there exist $\boldsymbol{P}>0, \boldsymbol{\Lambda} \geq 0$ and $\boldsymbol{T} \geq 0$ which satisfy LMI (10).

Remark : When we set $\boldsymbol{\Lambda}=0$, we obtain the LMI

$$
\left[\begin{array}{cc}
A^{T} P+P A & P B+T \\
B^{T} P+T & -2 T
\end{array}\right]<0
$$

which can be interpreted as a condition for the existence of a quadratic Lyapunov function $V(\boldsymbol{x})=\boldsymbol{x}^{\boldsymbol{T}} \boldsymbol{P} \boldsymbol{x}$ for Lur'e system.

To apply Theorem 1, the closed system (6) should be transformed into Lur'e system (8). (14)

First, we divide (6) into the linear and the nonlinear part as

$$
x^{(n)}-\widehat{\boldsymbol{a}}^{T} \cdot \boldsymbol{x}=\boldsymbol{a}_{N}(t)^{T} \cdot \boldsymbol{x}
$$

Then, the differential equation (14) can be represented by the state-space equation of (15).

$$
\begin{gathered}
\dot{\boldsymbol{x}}=\overline{\boldsymbol{A}} \boldsymbol{x}+\overline{\boldsymbol{B}} \overline{\boldsymbol{p}} \\
\overline{p_{i}}(t)=\overline{\phi_{i}}\left(x_{i}(t)\right), \quad i=1, \cdots, n
\end{gathered}
$$

where,

$$
\overrightarrow{\boldsymbol{A}}=\left[\begin{array}{ccccc}
0 & 1 & 0 & \cdots & 0 \\
0 & 0 & 1 & \cdots & 0 \\
0 & 0 & 0 & \cdots & 1 \\
\widehat{a}_{1} & \widehat{a}_{2} & \vdots & & \\
\hat{a}_{3} & \cdots & \widehat{a_{n}}
\end{array}\right], \quad \bar{B}=\left[\begin{array}{ccccc}
0 & 0 & 0 & \cdots & 0 \\
0 & 0 & 0 & \cdots & 0 \\
0 & 0 & 0 & \cdots & 0 \\
& & \vdots & & \\
1 & 1 & 1 & \cdots & 1
\end{array}\right]
$$

and

$$
\overline{\phi_{i}}\left(x_{i}(t)\right)=a_{N i}(t) x_{i}(t), \quad i=1, \cdots, n
$$

The sector condition of $\bar{\phi}_{i}$ is

$$
\begin{gathered}
\alpha_{i} \sigma^{2} \leq \sigma \overline{\phi_{i}}(\sigma) \leq \beta_{i} \sigma^{2}, \quad i=1, \cdots, n \\
\text { where } \alpha_{i}=\min _{t}\left(a_{N j}(t)\right) \text { and } \\
\beta_{i}=\max _{t}\left(a_{N j}(t)\right) .
\end{gathered}
$$

The sector bounds $\alpha_{i}$ and $\beta_{i}$ can be obtained by the method in Appendix.

By substituting the equations (17) into (15) and (16), the general $\left[\alpha_{i}, \beta_{i}\right]$ sector condition for $\overline{\phi_{i}}(16)$ can be cast into the $[0,1]$ sector condition (9) for $\phi_{i}$. This substitution procedure is called 'loop transformation'.

$$
\begin{aligned}
& \bar{p}_{i}=\bar{\phi}_{i}\left(x_{i}(t)\right)=\left(\beta_{i}-\alpha_{i}\right) p_{i}+\alpha_{i} x_{i} \\
& p_{i}(t)=\phi_{i}\left(x_{i}(t)\right), \quad i=1, \cdots, n
\end{aligned}
$$

or in the matrix form.

$$
\begin{aligned}
\bar{p}= & M p+N q \\
\text { where } & M=\operatorname{diag}\left(\alpha_{1}, \cdots, \alpha_{n}\right) \text { and } \\
& N=\operatorname{diag}\left(\beta_{1}-\alpha_{1}, \cdots, \beta_{n}-\alpha_{n}\right)
\end{aligned}
$$

And the resulting Lur'e system of loop transformation can be expressed as (18)

$$
\begin{gathered}
\dot{\boldsymbol{x}}=\boldsymbol{A} \boldsymbol{x}+\boldsymbol{B} \boldsymbol{p} \\
p_{i}(t)=\phi_{i}\left(x_{i}(t)\right), \quad i=1, \cdots, n_{p}
\end{gathered}
$$

where $A=\bar{A}+\bar{B} N$ and $B=\bar{B} M$

$\phi_{i}\left(x_{i}(t)\right)=\frac{a_{N_{i}}(t)-\alpha_{i}}{\beta_{i}-\alpha_{i}} x_{i}(t), \quad i=1, \cdots, n$

Applying Theorem 1 to the transformed Lur'e system (18), we can derive the robust stability condition for the closed system (6), as in Theorem 2. 
Theorem 2 : The closed system (6) is robust stable in the sense of Lyapunov if the corresponding Lur'e system (18) of the closed system (6) satisfies Theorem 1.

Proof : The closed system (6) can be transformed into the corresponding Lur'e system (18) by the above-mentioned loop transformation. Therefore, the stability of Lur'e system (18) implies the robust stability of the closed system (6). Thus, if the corresponding Lur'e system (18) of the closed system (6) satisfies Theorem 1, then we can conclude that the closed system (6) is robust stable in the sense of Lyapunov.

\section{Conclusion}

In this paper, we have presented the LMI-based robust stability condition which can be solved numerically for the fuzzy feedback linearization regulator via Takagi-Sugeno fuzzy model. We implemented the fuzzy feedback linearization regulator based on Takagi-Sugeno fuzzy model and proposed the LMI-based robust stability condition.

\section{References}

[1] Slotine, J. E. and Li, W.: "Applied nonlinear control", Prentice-Hall, Englewood Cliffs, 1991

[2] Isidori, A.: "Nonlinear control systems", Springer- Ve rlag, Berlin, 1989

[3] Sugeno, M.: "Fuzzy control", Nikangoubyou- Shinnbu n-sha, Tokyo, 1988

[4] Wang, L.: "Adaptive fuzzy systems and control: design and stability analysis", Prentice-Hall, Englewood Cliffs, 1994

[5] Chen, B. S., Lee, C. H., Chang, Y. C.: " $H^{\infty}$ tracking design of uncertain nonlinear SISO systems : adaptiv fuzzy approach", IEEE Trans. Fuzzy Systems, 1996, 4(1) pp. $32-43$

[6] Fischle, K. and Schroder, D.: "An improved stable adaptive fuzzy control method", IEEE Trans. Fuzzy Systems, 1999, 7(1) pp.27-40

[7] Tsay, D. L., Chung, H. Y. and Lee, C. J.: "The adaptive control of nonlinear systems using the Sugeno-type of fuzzy logic", IEEE Trans. Fuzzy Systems, 1999, 7(2) pp. 225-229

[8] Kang, H. J., Kwon, C., Lee, C. H. and Park, M.: "Robust stability analysis and design method for the fuzzy feedback linearization regulator", IEEE Trans. Fuzzy Systems, 1998, 6(4) pp. 464-472

[9] Takagi, T., Sugeno, M.: "Fuzzy Identification of systems and its applications to modeling and control", IEEE Trans. Syst., Man, Cybern. 1985, 15(1) pp. 116-132

[10] Boyd, S.: "Linear matrix inequalities in systems and control theory", SIAM, Philadelphia, 1994

[11] Nesterov, Y., Nemirovsky, A.: "Interior-point polynomial methods in convex programming", SLAM, Philadelphia, 1994

[12] Wang, H. O., Tanaka, K., Grifiin, F. G.: "An approach to fuzzy control of nonlinear system: stability and design issues", IEEE Trans. Fuzzy Systems, 1996, 4(1) pp. $14-23$

[13] Tanaka, K., Ikeda, T., Wang, H. O.: "Robust stabilization of a class of uncertain nonlinear systems via fuzzy control: quadratic stabilizability, $H^{\infty}$ control theory, and linear matrix inequalities", IEEE Trans. Fuzzy Systems, 1996, 4(1) pp. 1-13

[14] Taniguchi, T., Tanaka, K., Yamafugi, K. and Wang, H. O.: "A new PDC for fuzzy reference models", FUZZ-IEEE'1999, Aug. 1999, Seoul, Korea, pp.898-903

[15] Lam, H. K., Leung, F. H. F and Tam, P. K. S.: "An improved stability analysis and design of fuzzy control systems", FUZZ-IEEE'1999, Aug. 1999, Seoul, Korea, pp. $430-433$,

[16] Kim, E., Kang, H. J., and Park, M.: "Numerical stability analysis of fuzzy control systems via quadratic programming and linear matrix inequalities", IEEE Trans. Fuzzy Systems, 1999, 29(4) pp. 333-346

[17] Lur'e, A. I.: "Some Nonlinear problems in the theory of automatic control", H. M. Stationery Off., London, 1957

\section{Appendix}

In the followings, we need the basic assumption that

$$
\sum_{i=1}^{k} h_{i}(x(t))=1 \text { and } \max _{x} h_{i}(x(t))=1 .
$$

The maximum and minimum sector bounds of $a_{N j}(t)$ can be computed from (25) and (26)

$$
\begin{aligned}
& =\max _{t}\left\{\sum_{i=1}^{r} h_{i}(x(t)) \Delta a_{i j}(t)\right\} \\
& +\max _{t}\left\{\frac{\sum_{i=1}^{L} h_{i}(x(t)) \Delta b_{i}(t)}{\sum_{i=1}^{r} h_{i}(x(t)) b_{i}} \cdot \sum_{i=1}^{r} h_{i}(x(t)) e_{i j}\right\} \\
& \min _{t}\left(a_{N j}(t)\right)=\min _{t}\left\{\sum_{i=1}^{r} h_{i}(x(t)) \Delta a_{i j}(t)\right\} \\
& +\min _{t}\left\{\frac{\sum_{i=1}^{r} h_{i}(x(t)) \Delta b_{i}(t)}{\sum_{i=1}^{r} h_{i}(x(t)) b_{i}} \cdot \sum_{i=1}^{r} h_{i}(x(t)) e_{i j}\right\}
\end{aligned}
$$

where $e_{i j}=\widehat{a_{j}}-a_{i j}$

The second terms of (25) and (26) can be computed using the following property.

$$
\left.\min _{i}\left(\Delta a_{i j}(t)\right) \leq \sum_{i=1}^{r} h_{i}(x(t)) \Delta a_{i j}(t)\right) \leq \max _{i}\left(\Delta a_{i j}(t)\right)
$$

The third terms of (25) and (26) can be computed from (28) and (29)

$$
\max _{t}\left\{\frac{\sum_{i=1}^{x} h_{i}(x(t)) \Delta b_{i}(t)}{\sum_{i=1}^{x} h_{i}(x(t)) b_{i}} \cdot \sum_{i=1}^{k} h_{i}(x(t)) e_{i j}\right\}
$$




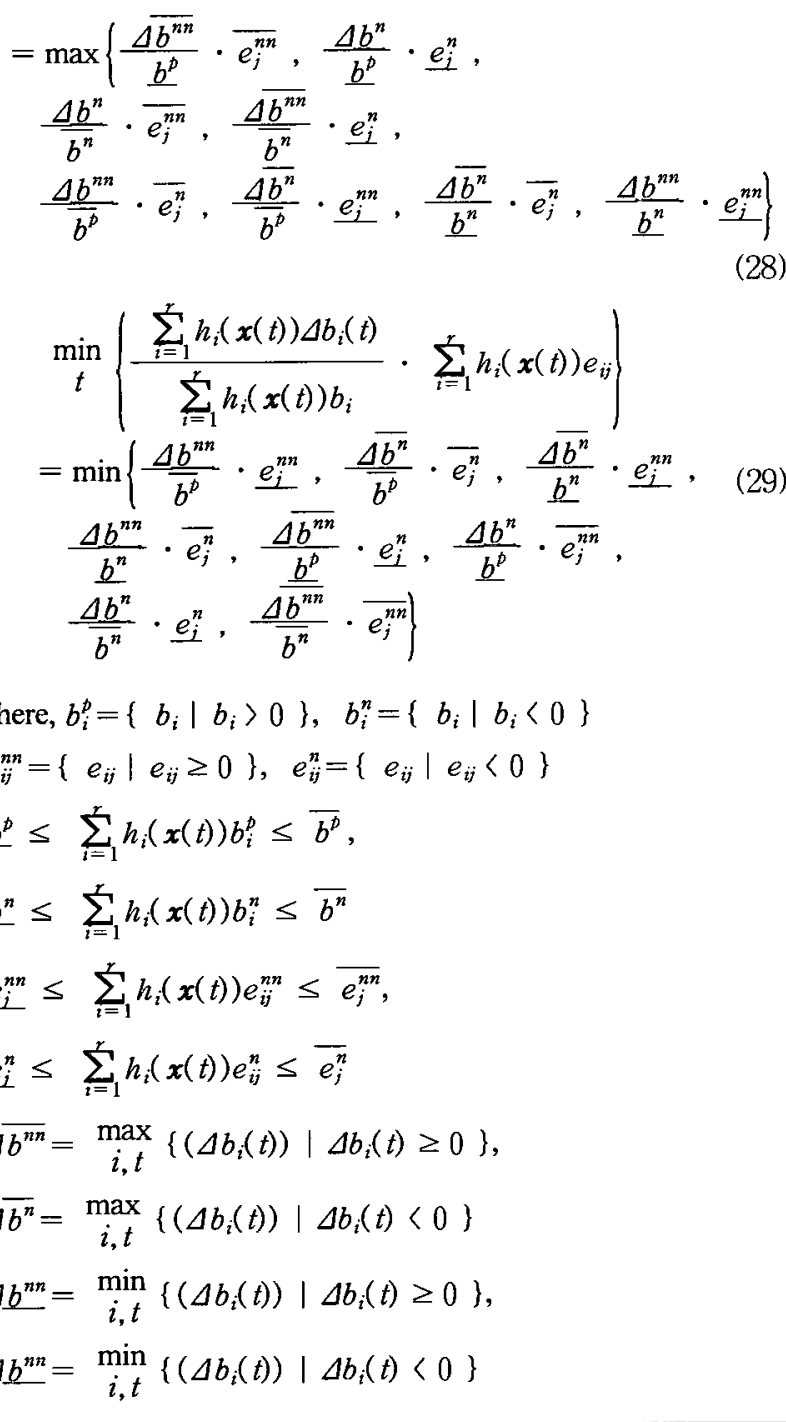

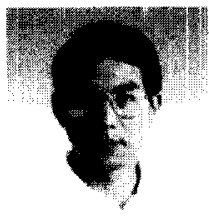
fuzzy control system, nonlinear control, robotics, and digital watermark.

Phone : $+82-2-2123-2868$

Fax : +82-2-312-2333

E-mail : chlee@kist.re.kr

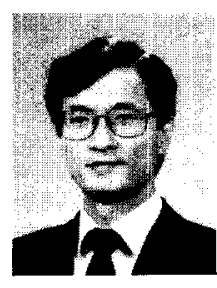

Mignon Park

received the B.S. and M.S. degrees in electronics from Yonsei University, Seoul, Korea, in 1973 and 1977, respectively, and the $\mathrm{Ph}$. D. degree from the University of Tokyo, Tokyo, Japan, in 1982. Since 1982, he has been a Professor in the Electrical and Computer Department of Yonsei University. His research interests include fuzzy control and application engineering, robotics, and fuzzy biomedical system, etc.

Phone : +82-2-2123-2868

Fax : +82-2-312-2333

E-mail : mignpark@yonsei.ac.kr

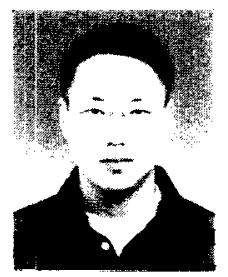

\section{Chang-Woo Park}

was born in Seoul, Korea, in 1973. He received the B.S. degree in electron:cs from Korea University and M.S. degree in electronics from Yonsei University, Seoul, Korea, in 1997 and 1999, respectively. He is currently working toward the Ph.D. degree in electrical and electronic engineering at Yonsei University, Seoul, Korea. His current research interests include fuzzy control theory, nonlinear control theory and robot vision system.

\footnotetext{
Phone : $+82-2-2123-2868$

Fax : +82-2-312-2333

E-mail : cwpark@yeics.yonsei.ac.kr
} 